\title{
FOCUSED R \\ Interstitial Pneumonia with Autoimmune Features
}

\author{
Bridget A. Graney ${ }^{1}$ and Aryeh Fischer ${ }^{1,2}$ \\ ${ }^{1}$ Division of Pulmonary Sciences and Critical Care Medicine and ${ }^{2}$ Division of Rheumatology, University of Colorado Anschutz Medical \\ Campus, Aurora, Colorado
}

\begin{abstract}
Interstitial pneumonia with autoimmune features (IPAF) is a research classification proposed by the European Respiratory Society/American Thoracic Society Task Force on Undifferentiated Forms of Connective Tissue Disease-associated Interstitial Lung Disease as an initial step to uniformly define, identify, and study patients with interstitial lung disease (ILD) who have features of autoimmunity, yet fall short of a characterizable connective tissue disease. Since its publication in July 2015, there has been substantial interest in IPAF. Centers from around the world have published their findings of retrospectively identified cohorts of patients who fulfill IPAF criteria, suggestions for modification of the criteria have been offered, and patients who fulfill IPAF criteria are being included as a subset in the ongoing phase II multicenter unclassifiable ILD treatment trial with pirfenidone. The
\end{abstract}

IPAF designation represents an important first step toward studying and furthering our understanding of the natural history of this cohort of patients with ILD using uniform nomenclature and a standardized set of criteria. Prospective evaluations and, ideally, interdisciplinary and multicenter collaborations will inform best practices for treatment and management and will guide future refinement to the IPAF criteria. This review focuses on the relevant background that led to the development of IPAF, summarizes the proposed criteria, discusses cohort studies of patients with IPAF published to date and what they have taught us about the IPAF phenotype, and offers insights into future directions in this arena.

Clinical trial registered with www.clinicaltrials.gov (NCT03099187).

Keywords: idiopathic interstitial pneumonias; connective tissue diseases; interstitial lung diseases; pulmonary fibrosis

(Received in original form August 20, 2018; accepted in final form January 29, 2018)

Supported by the Reuben M. Cherniak Fellowship Award at National Jewish Health (B.A.G.).

Correspondence and requests for reprints should be addressed to Aryeh Fischer, M.D., Divisions of Rheumatology, Pulmonary Sciences, and Critical Care Medicine, University of Colorado School of Medicine, 12631 East 17th Avenue, Academic Office Building One, Aurora, CO 80045. E-mail: aryeh.fischer@ucdenver.edu.

CME will be available for this article at http://www.atsjournals.org.

Ann Am Thorac Soc Vol 16, No 5, pp 525-533, May 2019

Copyright (C) 2019 by the American Thoracic Society

DOI: 10.1513/AnnalsATS.201808-565CME

Internet address: www.atsjournals.org

Interstitial lung disease (ILD) refers to a heterogeneous group of diffuse parenchymal lung disorders characterized by varying degrees of inflammation and/or fibrosis of the pulmonary interstitium, ultimately resulting in parenchymal damage. The ILDs are classified by specific patterns of clinical, radiographic, and histopathologic features and are broadly grouped into those considered idiopathic and those with an identifiable etiology. The most commonly identified causes of ILD are environmental or occupational exposures to organic or inorganic dusts, connective tissue disease (CTD), and drug- or radiation-induced lung injury (Figure 1).
The diagnosis of an idiopathic interstitial pneumonia (IIP), of which idiopathic pulmonary fibrosis (IPF) is the most common, requires the exclusion of known causes of ILD. As per international guidelines (1), patients newly diagnosed with ILD should undergo evaluation including clinical history and examination, pulmonary function testing (PFT), high-resolution computed tomography (HRCT) scan, surgical lung biopsy if indicated, and multidisciplinary discussion between experienced clinicians, radiologists, and pathologists to provide the most accurate diagnosis and distinguish an IIP from ILD with an identifiable etiology.
One of the most common underlying causes of ILD is CTD, which is identified in up to $30 \%$ of new ILD diagnoses (2). Because of the high prevalence of CTD in patients with newly diagnosed ILD, current guidelines recommend excluding the presence of an underlying CTD (1). Distinguishing CTD-associated ILD (CTD-ILD) from an IIP, particularly IPF, is important, because CTD-ILD has a more favorable prognosis, and the available therapeutic options differ significantly (3-5). Despite current recommendations and the importance of an accurate diagnosis to guide treatment decisions and inform patients of their prognosis, there is no standardized approach to assess for CTD. 


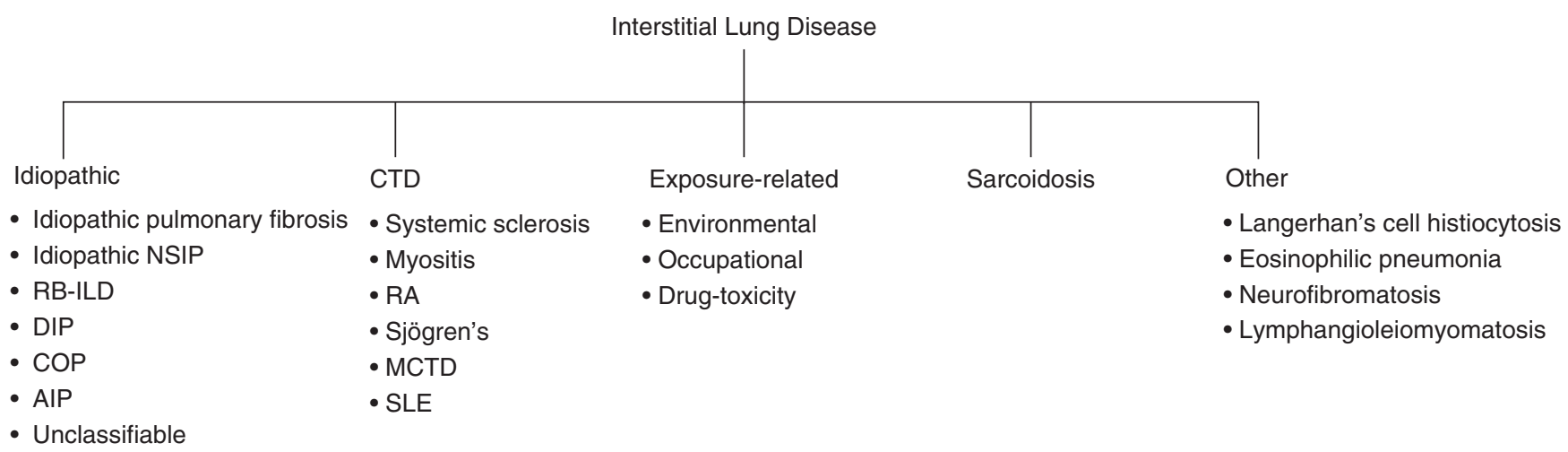

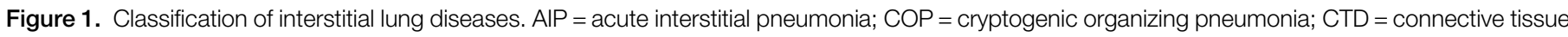
disease; DIP = desquamative interstitial pneumonia; MCTD = mixed connective tissue disease; NSIP = nonspecific interstitial pneumonia; RA = rheumatoid arthritis; RB-ILD = respiratory bronchiolitis-associated interstitial lung disease; SLE = systemic lupus erythematosus. Adapted by permission from Reference 52.

Moreover, the type and extent of evaluation varies by center, is often practitioner dependent, and is impacted by the composition of the multidisciplinary team-particularly regarding rheumatologic engagement.

Practically speaking, despite extensive efforts to identify occult CTD in patients with IIP, in many instances features suggesting background autoimmunity are identified, yet the patient may not have a characterizable CTD. Take, for example, a 45 -year-old woman with a pattern of usual interstitial pneumonia (UIP) by HRCT that is confirmed by surgical lung biopsy. There are no extrathoracic symptoms or signs of a CTD. Serologically, there is an isolated positive anti-SS-A antibody, and further review of her lung histopathology reveals lymphoid follicles with germinal center formation. This clinical scenario appears to represent an "autoimmune ILD" on the basis of the integration of clinical and pathologic data, yet on rheumatologic assessment she is not found to have any characterizable CTD. How should this patient be classified-and, more importantly, how does such classification impact the therapeutic approach to her ILD? Should she be considered as having IPF and treated with an antifibrotic medication or as CTD-ILD and treated with immunosuppression?

It is not uncommon to have discord among specialists around how to classify such patients, and these differences can translate to disagreements over therapeutic strategy. To some extent, this scenario highlights the ongoing interdisciplinary divide that exists between pulmonary medicine and rheumatology, with the latter group often taking the lead on developing CTD classification criteria. Indeed, other than for systemic sclerosis, the presence of ILD is not a feature in any of the classification schemes for other CTDs. As a result, because a patient lacks the extrathoracic "autoimmune" features that, in aggregate, meet current diagnostic criteria for a specific CTD, she is not considered to have CTD by rheumatologic standards.

Though reliable determinants of prevalence are lacking, there is recognition that it is common to encounter patients with IIP who have subtle features suggestive of an autoimmune etiology and yet do not have a characterizable CTD (6). The terms "undifferentiated CTD" (UCTD), "lungdominant CTD," or "autoimmune-featured ILD" have all been used to describe such individuals, and each term has been associated with unique and differing sets of criteria (7-9). These sets of criteria are different enough that research studies using one set of criteria are not likely to be applicable to cohorts from centers using other sets of criteria. Assayag and colleagues (10) applied the differing criteria of UCTD (a broader form proposed by Kinder and colleagues [8] and a narrower form proposed by Corte and colleagues [3]), lung-dominant CTD, and autoimmunefeatured ILD to a cohort of 119 patients with IIP evaluated in a tertiary ILD referral program and found that $56 \%$ of patients fulfilled criteria for at least one of these designations, but only $18 \%$ fulfilled all sets. Application of the broadest, least-specific criteria (8) captured $41 \%$ of the cohort, but the narrowest, most specific (3) of the four only encompassed $21 \%$ (10). This study convincingly illustrated that a uniform definition and nomenclature was needed to study patients with suggestive forms of autoimmune ILD, particularly given the fact that these patients are often shared among specialties.

In an effort to build consensus and interdisciplinary collaboration, the American Thoracic Society and the European Respiratory Society supported an international working group on undifferentiated forms of CTD-ILD, consisting of an international, multidisciplinary panel including investigators from the centers that had put forth the previous criteria and terminology of suggestive forms of CTD-ILD (3, 7-9). The primary objective of this task force was to develop consensus surrounding the nomenclature and classification of patients with IIP and features of autoimmunity without a characterizable CTD (Figure 2). The task force published a research statement (11) outlining the "interstitial pneumonia with autoimmune features" (IPAF) concept and nomenclature along with a set of classification criteria (Table 1) to serve as a platform for future research of this IIP subset.

\section{Review of the IPAF Criteria}

The term "interstitial pneumonia with autoimmune features" was chosen to highlight the distinct nature of this subset of 


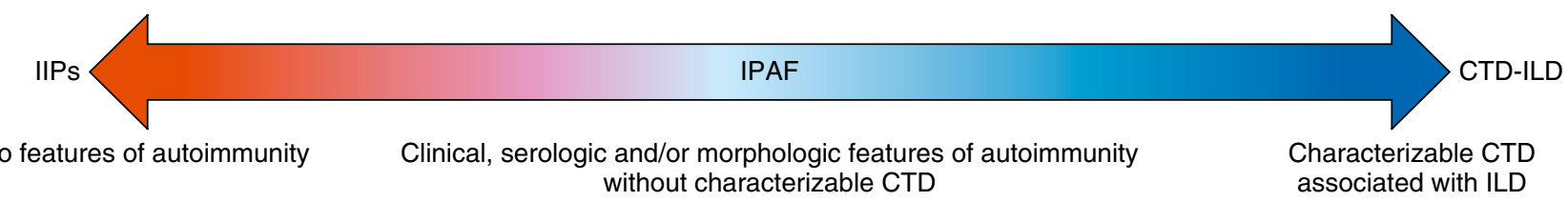

Figure 2. Continuum on which interstitial pneumonia with autoimmune features (IPAF) sits between the idiopathic interstitial pneumonias and defined connective tissue disease-related interstitial lung diseases (CTD-ILDs). IIP = idiopathic interstitial pneumonia.

ILD. The term "connective tissue disease" was specifically avoided, because of concerns that such labeling gives a false impression that these individuals are predetermined as having a CTD. Several a priori requirements must be fulfilled for the classification of IPAF: individuals must have evidence of interstitial pneumonia by HRCT imaging and/or by surgical lung biopsy, known causes for interstitial pneumonia must have been excluded after a thorough clinical evaluation, and patients do not meet criteria for a characterizable CTD. The classification criteria are then organized around three central domains: a clinical domain consisting of specific extrathoracic features, a serologic domain consisting of specific circulating autoantibodies, and a morphologic domain consisting of specific chest imaging features, histopathologic features, or pulmonary physiologic features. To be classified as IPAF, the individual must meet all of the a priori requirements and have at least one feature from at least two of the three domains (11).

\section{Clinical Domain}

The clinical domain consists of extrathoracic features that suggest an underlying CTD, but, on their own, none are diagnostic of a specific CTD. The features include distal digital fissuring ("mechanic hands"), digital tip ulceration, inflammatory arthritis or polyarticular morning joint stiffness lasting more than 60 minutes, palmar telangiectasia, Raynaud phenomenon, unexplained digital edema, and unexplained fixed rash on the digital extensor surfaces (Gottron's sign). Lessspecific features that were included in prior classification schemes (e.g., joint pain, myalgias, weight loss, photosensitivity, and sicca symptoms) were not included. The task force suggested that, ideally, the presence of clinical features should be assessed on physical examination by experienced practitioners and that self-reported symptoms should not be used alone to identify clinical domain features.

\section{Serologic Domain}

The serologic domain consists of autoantibodies that are strongly associated with CTD and exclude nonspecific markers of inflammation, such as erythrocyte sedimentation rate or $\mathrm{C}$-reactive protein. For the lessspecific antibodies-antinuclear antibody (ANA) and rheumatoid factor-sufficiently high titers are required to fulfill the serologic domain criteria. ANA positivity with a diffuse homogeneous or speckled pattern at low titer can be found in healthy control subjects, healthy elderly patients (12), and patients with IPF (13), thus prompting a higher titer threshold to meet criteria. ANA of any titer with nucleolar or centromere staining pattern meets the serologic domain criteria, as these patterns are strongly associated with systemic autoimmune disease. ANA should be measured by indirect immunofluorescence for accurate results.

\section{Morphologic Domain}

The morphologic domain consists of three subdomains: radiographic patterns by HRCT, histopathologic features from surgical lung biopsy, and multicompartment features (i.e., additional thoracic involvement). If any one item from the three subdomains is present, the criteria for the morphologic domain are fulfilled.

The radiographic subdomain describes patterns from HRCT that include nonspecific interstitial pneumonia (NSIP), organizing pneumonia (OP), NSIP with OP overlap, and lymphocytic interstitial pneumonia (LIP). NSIP is the most common pattern on HRCT in patients with CTD-ILD (14), and the presence of NSIP, OP, or LIP patterns on HRCT poses additional consideration of an underlying autoimmune process. Although UIP pattern on HRCT is the most common pattern in rheumatoid arthritis-associated ILD (15) and is also encountered in other CTD-ILDs (16), it was not given the same weight as the other IIP patterns because it is less specific for CTD.

The histopathologic pattern on surgical lung biopsy is the second subdomain within the morphologic domain. The features included in this subdomain-NSIP, OP, NSIP with OP, and LIP-are highly associated with the presence of a CTD (17). In addition to the overall histopathologic pattern on surgical lung biopsy, secondary features of interstitial lymphoid aggregates with germinal centers and diffuse lymphoplasmacytic infiltration are included in this subdomain.

Despite shared nomenclature, HRCT patterns do not correlate well with histopathologic patterns on surgical lung biopsy, with the exception of UIP (18-22). This lack of correlation for NSIP and OP patterns between HRCT and histopathology informs the rationale for including separate HRCT and histopathologic subdomains within the morphologic domain. Similar to the radiographic subdomain, UIP pattern on surgical lung biopsy was not included in the criteria because of its lack of association with an increased likelihood of CTD.

It is worth emphasizing that the presence of a radiologic or histopathologic UIP pattern does not exclude an IPAF designation but, unlike NSIP, OP, or LIP patterns, there is no morphological "credit" given to the UIP pattern. Thus, a patient with UIP pattern on HRCT or surgical lung biopsy still meets the IPAF definition by the same criterion: meeting all a priori requirements and having at least one feature from two of the three domains: clinical, serologic, or morphologic.

The final subdomain of the morphologic domain is the multicompartment designation, which includes unexplained pleural or pericardial thickening or effusions, 
Table 1. Classification criteria for interstitial pneumonia with autoimmune features

1. Presence of an interstitial pneumonia by HRCT or SLB and

2. Exclusion of alternative etiologies and

3. Does not meet criteria for a defined CTD and

4. At least one feature from at least two of the following domains:

\section{A. Clinical domain}

1. Distal digital fissuring (i.e., "mechanic hands")

2. Distal digital tip ulceration

3. Inflammatory arthritis or polyarticular morning joint stiffness $\geqslant 60 \mathrm{~min}$

4. Palmar telangiectasia

5. Raynaud phenomenon

6. Unexplained digital edema

7. Unexplained fixed rash on the digital extensor surfaces (Gottron sign)

\section{B. Serologic domain}

1. ANA $\geqslant 1: 320$ titer, diffuse, speckled, homogeneous patterns or

a. ANA nucleolar pattern (any titer) or

b. ANA centromere pattern (any titer)

2. Rheumatoid factor $\geqslant 2 \times$ upper limit of normal

3. Anti-CCP

4. Anti-dsDNA

5. Anti-Ro (SS-A)

6. Anti-La (SS-B)

7. Anti-ribonucleoprotein

8. Anti-Smith

9. Anti-topoisomerase (Scl-70)

10. Anti-tRNA synthetase (e.g., Jo-1, PL-7, PL-12; others are: EJ, OJ, KS, Zo, tRS)

11. Anti-PM-Scl

12. Anti-MDA-5

\section{Morphologic domain}

1. Suggestive radiology patterns by HRCT:
a. NSIP
b. OP
c. NSIP with OP overlap
d. LIP

2. Histopathology patterns or features by surgical lung biopsy:

a. NSIP

b. OP

c. NSIP with OP overlap

d. LIP

e. Interstitial lymphoid aggregates with germinal centers

f. Diffuse lymphoplasmacytic infiltration (with or without lymphoid follicles)

3. Multicompartment involvement (in addition to interstitial pneumonia):

a. Unexplained pleural effusion or thickening

b. Unexplained pericardial effusion or thickening

c. Unexplained intrinsic airways disease (by PFT, imaging or pathology)

d. Unexplained pulmonary vasculopathy

Definition of abbreviations: ANA = antinuclear antibody; CTD = connective tissue disease; HRCT = high-resolution computed tomography; LIP = lymphocytic interstitial pneumonia; NSIP = nonspecific interstitial pneumonia; OP= organizing pneumonia; PFT = pulmonary function testing; SLB = surgical lung biopsy. *Includes airflow obstruction, bronchiolitis or bronchiectasis.

Adapted by permission from Reference 11.

intrinsic airways disease, or pulmonary vasculopathy. Patients with CTD often demonstrate involvement of other pulmonary structures, including airways, pulmonary vasculature, and the pleura with varying degrees of frequency with respect to the underlying disease (Table 2$)(4,23)$. The current research statement does not provide definitions for determining the presence of "unexplained" multicompartment involvement with respect to airways, pleural, or vascular disease, or guidelines to interpret the presence of "diffuse" lymphoplasmacytic infiltration or lymphoid aggregates with germinal centers on biopsy. Therefore, identifying features of the multicompartment subdomain are left to the discretion of the individual clinician or investigator.

Table 2. Connective tissue disease-associated pulmonary manifestations

\begin{tabular}{lcccccc}
\hline & SSc & PM/DM & RA & Primary Sjögren & MCTD & SLE \\
ILD & +++ & +++ & ++ & ++ & ++ & + \\
Airways & - & - & ++ & ++ & + & + \\
Pleural & - & - & - & - & + & +++ \\
Vascular & +++ & + & - & - & + & ++ \\
DAH & - & - & - & + & + & + \\
\hline
\end{tabular}

Definition of abbreviations: $\mathrm{DAH}=$ diffuse alveolar hemorrhage; ILD = interstitial lung disease; MCTD = mixed connective tissue disease; PM/DM = polymyositis/dermatomyositis; RA = rheumatoid arthritis; SLE = systemic lupus erythematosus; SSc = systemic sclerosis.

The number of + signs indicates relative prevalence of each manifestation. Adapted by permission from Reference 4.

\section{Retrospectively Identified Cohorts with IPAF}

Since publication of the European Respiratory Society/American Thoracic Society IPAF research statement, a number of studies have retrospectively identified and described cohorts of patients with ILD who fulfill IPAF criteria (Table 3). It is important to note that each of these cohorts was identified retrospectively and is impacted by the methodologic limitations of cohort identification and selection.

Oldham and colleagues (24) identified 422 patients with either IIP or UCTD from their ILD database, and 144 (34\%) met IPAF criteria. The mean age of the IPAF group was 63.2 years, with a majority being female (52\%) and former smokers (55\%). The most common clinical feature was Raynaud phenomenon (27.8\%), and the most common serologic feature was ANA positivity (77.6\%). Although the most common morphologic domain feature was NSIP pattern by HRCT (31.9\%), the majority of the cohort demonstrated a UIP 
Table 3. Comparison of retrospectively identified interstitial pneumonia with autoimmune features cohorts

\begin{tabular}{|c|c|c|c|c|c|c|c|}
\hline & $\begin{array}{l}\text { Oldham et al. } \\
\qquad(24)\end{array}$ & $\begin{array}{l}\text { Chartrand et al. } \\
\text { (27) }\end{array}$ & $\begin{array}{c}\text { Ahmad et al. } \\
\text { (29) }\end{array}$ & $\begin{array}{l}\text { Ito et al. } \\
\text { (31) }\end{array}$ & $\begin{array}{l}\text { Dai et al. } \\
\text { (32) }\end{array}$ & $\begin{array}{l}\text { Yoshimura et al. } \\
\text { (34) }\end{array}$ & $\begin{array}{l}\text { Kelly and } \\
\text { Moua (35) }\end{array}$ \\
\hline Patients, $n$ & 144 & 56 & 57 & 98 & 177 & 32 & 101 \\
\hline Age, yr, mean $\pm S D$ & $63.2 \pm 11$ & $54.6 \pm 10.3$ & $64.4 \pm 14$ & $67.5 \pm 9$ & $67.6 \pm 8.6$ & $63.4 \pm 12.6$ & $56.9 \pm 14.2$ \\
\hline Female & 52.1 & 71.4 & 49.1 & 58.2 & 55.9 & 40.6 & 39 \\
\hline Ever-smoker & 54.9 & 32.1 & 34 & 38.8 & 19.2 & 56.2 & 31 \\
\hline Clinical & 49.3 & 62.5 & 47.3 & NR & 20.3 & 53.1 & NR \\
\hline Serologic & 91.7 & 91.1 & 93 & $100^{*}$ & 92.1 & 71.9 & NR \\
\hline Morphologic & 85.4 & 98.2 & 78.9 & $100^{\dagger}$ & 95.5 & 96.9 & NR \\
\hline Clinical and serologic & 14.6 & 2 & NR & NR & NR & 3.1 & 4 \\
\hline $\begin{array}{l}\text { Clinical and } \\
\text { morphologic }\end{array}$ & 8.3 & 9 & NR & NR & NR & 28.1 & 14 \\
\hline $\begin{array}{l}\text { Serologic and } \\
\text { morphologic }\end{array}$ & 50.7 & 37.5 & NR & 100 & NR & 46.9 & 26 \\
\hline All three domains & 26.4 & 52 & NR & NR & NR & 21.9 & 56 \\
\hline UIP by HRCT & 54.6 & 8.9 & 28 & 0 & 4.5 & NR & NR \\
\hline Underwent SLB, $n(\%)$ & $83(57.6)$ & $36(64.3)$ & $16(28.1)$ & $17(17.3)$ & $0^{\ddagger}$ & $22(68.8)$ & $51(50.5)$ \\
\hline UIP on SLB, $n(\%)$ & $61(73.5)$ & $8(22.2)$ & $3(18.8)$ & $3(17.6)$ & - & - & $12(23.5)$ \\
\hline \multicolumn{8}{|l|}{ Treatment } \\
\hline Corticosteroids & 32.2 & 81.8 & 67.9 & 17.3 & 72.3 & 59.4 & NR \\
\hline Antifibrotic & NR & NR & 5.4 & 2 & NR & 25 & NR \\
\hline \multicolumn{8}{|l|}{ Outcome } \\
\hline Death & 39.6 & 0 & 12.3 & 27.6 & 19.8 & NR & 28 \\
\hline Lung transplant & 10.8 & NR & $\mathrm{NR}$ & NR & NR & NR & NR \\
\hline
\end{tabular}

Definition of abbreviations: HRCT = high-resolution computed tomography; NR = not reported; NSIP = nonspecific interstitial pneumonia; OP = organizing pneumonia; SD = standard deviation; SLB = surgical lung biopsy; UIP = usual interstitial pneumonia pattern.

Data presented as percentage unless otherwise stated.

${ }^{*}$ Based on study design, inclusion criterion was positive serological evaluation.

${ }^{\dagger}$ Based on reported HRCT findings of NSIP, OP, or NSIP + OP in 98 of 98 subjects.

${ }^{\ddagger}$ All histopathology from transbronchial biopsies.

pattern on HRCT $(54.6 \%)$ and on surgical lung biopsy (61 of 83 patients biopsied, $73.5 \%)$. Twenty-six percent of patients met criteria in all three domains.

Survival analysis of the Oldham cohort demonstrated that those meeting IPAF criteria had slightly better outcomes than IPF but worse than those with CTD-ILD (24). After stratifying for the presence of UIP pattern on HRCT or surgical lung biopsy, non-UIP-IPAF had similar survival to CTD-ILD $(P=0.45)$, whereas UIP-IPAF demonstrated similar survival to IPF $(P=0.51)$. Predictors of increased mortality after multivariate analysis included age and diffusing capacity of the lung for carbon monoxide $\left(\mathrm{DL}_{\mathrm{CO}}\right)$, leading the authors to conclude that the Gender, Age, Physiology (GAP) scoring system (25), validated for mortality prediction in IPF, may be useful in IPAF as well. In a follow-up study of this cohort, Chung and colleagues focused on imaging predictors of survival and identified worse survival for subjects with IPAF when concomitant honeycombing or pulmonary artery enlargement is present (26).
Chartrand and colleagues (27) characterized a cohort of 56 patients meeting IPAF criteria. The cohort was identified from a group of patients who were all determined by the treating physician to have an autoimmune basis for their ILD, but without a characterizable CTD or alternative identifiable etiology. This cohort was slightly younger than that in the study by Oldham and colleagues (24), with a mean age of 55 years, and had a greater predominance of females $(71 \%)$ and never-smokers (68\%) (27). Similar to the Oldham cohort, Raynaud phenomenon (39\%) and ANA positivity (48\%) were the most common clinical and serologic domain features, respectively. Fifty-five of the 56 patients (98\%) fulfilled IPAF morphologic criteria, as compared with $85 \%$ in the Oldham cohort. By HRCT, the predominant pattern was NSIP (57\%), followed by the combination of NSIP and OP (18\%). Of the 36 patients who underwent surgical lung biopsy, 12 (33\%) had NSIP and $8(22 \%)$ had UIP.

A majority of patients (52\%) in the Chartrand cohort met criteria in all three domains. This was likely driven by the greater number of patients with identified clinical features as compared with the Oldham cohort $(62.5 \%$ in the Chartrand cohort vs. $49.3 \%$ in the Oldham cohort). Each patient in the Chartrand cohort was primarily under the care of a rheumatologist, which may explain the increase in identified clinical features. Furthermore, $36 \%$ of the cohort had a positive tRNA synthetase antibody, and the authors acknowledged that although the patients lacked other features of myositis spectrum, some could consider these individuals to have partial presentation of the antisynthetase syndrome (28).

Ahmad and colleagues (29) published data from a European cohort, comparing those with IPAF to those with IPF. Patients were identified consecutively over 3 years from a hospital discharge database searching for those with IIP or CTD-ILD. Of 778 patients screened, 156 (20.1\%) had IPF, 167 (21.5\%) had CTD-ILD, and 57 (7.3\%) met criteria for IPAF. Similar to the Oldham cohort, patients had a mean age of 64 years but with very slight male predominance (50.9\%). Raynaud phenomenon (74\%) and ANA positivity (82\%) were again the most common clinical and serologic features. 
Clinical features, similar to the Oldham cohort, were identified in $47 \%$ of patients. Morphologically, 53\% had NSIP pattern and $28 \%$ had UIP pattern on HRCT. Sixteen patients of the IPAF group underwent surgical lung biopsy; NSIP pattern was identified in five $(31 \%)$ and UIP pattern in three (19\%).

Overall survival at 1 year was not different between those with IPAF (83.6\%) and IPF $(94.8 \%)(P=0.05)$, and among the IPAF cohort, UIP pattern on HRCT was not associated with worse survival. One critique of this cohort was that some of the patients ( $n=13,23 \%)$ had abnormal nailfold capillaroscopy demonstrating giant capillaries. On the basis of this finding, some would consider this subset to have an early form of systemic sclerosis (30), which would exclude them from an IPAF designation.

Ahmad and colleagues proposed a novel "IPAF score" indicating the total number of IPAF criteria that were met in any of the three domains. In their cohort, the mean score was 3.5 , with a maximum score of 9 in one patient. Although the IPAF score was not associated with survival in this study, hypothetically, a higher "IPAF score" may be associated with progression to a defined CTD.

Ito and colleagues (31) retrospectively identified a cohort of 98 patients who fulfilled IPAF criteria. The primary aim of this study was to determine prognostic factors of the serologic and morphologic IPAF domains. Therefore, the cohort was derived by screening a database of 1,057 subjects with IIP first for positive serologies $(n=332)$; after excluding subjects for incomplete data or failure to meet the $a$ priori IPAF requirements, the group was narrowed to those meeting the morphologic domain by HRCT pattern. This search strategy derived the final cohort of 98 patients who were subsequently analyzed to identify prognostic factors.

The cohort had a mean age of 67.5 years and was predominantly female $(58.2 \%)$ and never-smokers (61.2\%). Some $64.3 \%$ of patients had NSIP pattern on HRCT. Their analysis demonstrated that increasing age and NSIP pattern on HRCT, as compared with NSIP with OP overlap or OP alone, were associated with shortened survival. A limitation to this cohort is that the search strategy used could plausibly miss patients with IIP who would have otherwise met IPAF criteria.
Dai and colleagues (32) retrospectively identified a Chinese cohort of 177 patients from an ILD database who fulfilled criteria for IPAF and compared them to a group of 1,252 patients with ILD who did not fulfill IPAF criteria. The IPAF cohort had a mean age of 60.2 years and was $55.9 \%$ female and $80.8 \%$ never-smokers. Raynaud phenomenon (12.9\%), ANA positivity (49.2\%), and NSIP on HRCT (61.6\%) were the most common features from the three domains. Interestingly, only $20.3 \%$ of the cohort had an identified clinical feature. Eight patients (4.5\%) had UIP pattern on HRCT. No patients underwent surgical lung biopsy. Multivariate analysis demonstrated age, smoking history, anti-RNP positivity, and OP pattern on HRCT as predictors for worsened survival.

Focusing on a subset of patients with chronic fibrotic interstitial pneumonia, Yoshimura and colleagues retrospectively applied the IPAF criteria to a cohort of 194 patients from their center, of whom 163 (84\%) had a clinical diagnosis of IPF (33). Thirty-two patients (16.5\%) met the criteria for IPAF. Patients with IPAF were significantly younger and included a higher proportion of women, never-smokers, and patients with NSIP compared with those without IPAF. Fulfilment of the IPAF criteria was an independent predictor of overall survival (95\% confidence interval, $0.017-0.952 ; P=0.045)$ and incidence of acute exacerbations (95\% confidence interval, 0.054-0.937; $P=0.040$ ) (33).

Finally, Kelly and Moua reviewed the charts of 101 patients whom they had defined as having UCTD-ILD and noted that the vast majority (91\%) also met criteria for IPAF (34). They too highlighted frequent clinical findings of Raynaud phenomenon, a positive ANA, and HRCT features suggestive of NSIP (34).

\section{Lessons Learned from Retrospective IPAF Cohorts}

The cohort studies described reflect the retrospective application of the IPAF criteria from single centers around the world. Each study is limited by referral bias, by methodologic limitations of cohort identification, and by how specifics of the IPAF criteria-in particular the morphologic domain-were applied. Despite these differences, several themes emerge: 1) Similar to ILD in general, and in particular the spectrum of CTD-ILD, there appears to be significant heterogeneity within the IPAF phenotype, which may have prognostic implications. Just like there is an appreciation for diverse lung injury patterns in characterizable CTD-ILD-and that fibrotic patterns may portend worse prognosis (35) - there may be similar heterogeneity among those who fulfill IPAF criteria. Furthermore, it is plausible to consider that, similar to IIPs (36), an underlying pattern of UIP among those who fulfill IPAF criteria may be associated with worse survival. Recognition of heterogeneity and identifying the important factors determining prognosis may prove valuable for patient education and management decisions. 2) Determination of clinical features is likely to be more reliably detected in prospective assessments geared toward their specific detection rather than by retrospective review or by patient report and may be optimized by direct rheumatologic engagement in the evaluation of these patients. 3) Revisions to the IPAF criteria are needed, particularly with respect to the morphologic domain. Because the IPAF criteria do not rigorously define how to characterize aspects of the morphology domain, individual providers, investigators, and studies define them and identify them differently.

One major advantage of IPAF is that uniform nomenclature has been adopted, prospective research studies from diverse programs are using similar classification criteria, data are being gathered to allow for anticipated refinement of the criteria in an evidence-based manner, and there is far more interdisciplinary engagement around this intersection of pulmonary and rheumatologic medicine.

\section{Future Directions}

The IPAF proposal represents an initial consensus classification scheme put forth by a relatively small panel of multidisciplinary international experts in the field. The task force acknowledged that the proposed classification scheme was a "first draft" and anticipated that revisions to the proposal would be needed when informed by data. The designation has spurred healthy interdisciplinary dialogue, and it has become clear that modifications are needed to refine the original classification scheme. Indeed, as acknowledged in a recent expert commentary, the IPAF criteria have an intrinsically changing structure and perhaps 
"the most important effect of these criteria is the identification of a gray zone of not well-defined rheumatology conditions" (37).

Numerous questions exist about the individual items within each domain. With respect to the clinical domain, a common question is if esophageal dysmotility should be included, given that this feature is common in patients with CTD. If included, how would this be defined-by esophageal manometry and/or degree of dilation by HRCT and/or barium esophagram? What about calls to broaden the clinical criteria in general by including nonspecific variables such as keratoconjunctivitis sicca or arthralgias? Should rheumatologic consultation be required to specifically assess for musculoskeletal features, particularly given the increased number of clinical features identified with specific rheumatologic involvement (27)? Should dermatologic consultation be required for cutaneous assessment? How are we to know where to start and where to stop? Undoubtedly, many of these aspects are based on collective expert opinion rather than driven by evidence per se. With the next iteration, other than expanding and diversifying the panel, a more systematic approach (e.g., Delphi exercise) and global input should be considered.

With respect to the serologic domain, there has been controversy around the exclusion of antineutrophil cytoplasmic antibody (ANCA) and the inclusion of anti-tRNA synthetase antibodies (38, 39). Should the serologic domain include ANCA along with proteinase- 3 and myeloperoxidase antibodies? Indeed, ANCA positivity in various forms of fibrotic interstitial pneumonia has been described in patients with or without features of systemic vasculitis $(40,41)$. Furthermore, controversy surrounds the inclusion of anti-tRNA synthetase antibodies, as this ultimately raises the question of the definition of "antisynthetase syndrome" and if the presence of ILD with a tRNA synthetase antibody is sufficient for this classification. According to the current idiopathic inflammatory myositis scheme (42), these two features alone would not meet criteria. However, in large measure as a result of interdisciplinary interests in IPAF and the relationship to antisynthetase syndrome, there is now an international effort to develop consensus classification criteria for antisynthetase syndrome (37). Furthermore, novel autoantibodies associated with ILD will continue to be identified and changes to the serologic domain will be needed.

The morphologic domain may remain the most challenging. Since the criteria were proposed, one common critique of the morphologic domain is the exclusion of UIP pattern on HRCT or surgical lung biopsy (43). Importantly, and as discussed above, the presence of a UIP pattern does not exclude a designation of IPAF. In the currently available IPAF literature, the frequency of UIP on HRCT and surgical lung biopsy has varied widely. Furthermore, outcomes for patients with IPAF and the presence of a UIP pattern (UIP-IPAF) have been variable in the published cohorts. In part this may be driven by how the retrospective cohorts were identified. Prospective studies will provide valuable data to inform the prevalence and outcomes of patients with UIP-IPAF.

Studies comparing CTD-ILD with UIP pattern on HRCT or surgical lung biopsy have consistently demonstrated improved survival as compared with idiopathic UIP (IPF) $(36,44-46)$, with the exception of rheumatoid arthritis (RA)-UIP, for which the data are conflicting $(45,47)$. These studies suggest that patients with an autoimmune-mediated basis to their lung disease have improved survival even with similar radiographic or histologic pattern of fibrosis, highlighting the importance of etiology in ILD natural history. As such, identifying and distinguishing between patients with truly idiopathic disease and UIP (i.e., the clinical diagnosis of IPF) from those with clinical, serologic, and/or morphologic features of autoimmune disease and UIP (i.e., UIP-IPAF) may have significant prognostic implications.

Furthermore, it is likely to alter management decisions. In the current era, the use of antifibrotic therapy is limited to those with IPF because clinical trials have shown benefit in this patient population (48, 49). Data from prospective clinical trials are needed to determine whether antifibrotic therapy has a similar role in the management of other cohorts with UIP pattern of lung injury, including those with IPAF or fibrotic ILD in general.

Ambiguity exists with the current IPAF criteria regarding the extent of germinal centers or lymphoplasmacytic infiltration on histopathology required to fulfill the morphological domain. One recent study (50) designated at least three germinal centers in any one low-power field and the presence of lymphocytes and 40 or more plasma cells in a high-power field as meeting criteria, but to date there has been no consensus on this definition.

There need to be more uniform specifications regarding how to define multicompartment involvement in patients beyond "unexplained airways disease or unexplained pulmonary vasculopathy." How should airways disease be defined-by PFT, imaging, and/or histopathology? And does any degree of smoking history preclude the designation of airways disease as "unexplained"? Similarly, can pulmonary vasculopathy be defined by suggestive, noninvasive tests such as PFT

(i.e., disproportionately decreased $\mathrm{DL}_{\mathrm{CO}}$ ) or echocardiography, or is invasive testing with right-heart catheterization required? Is the presence of vasculopathy on histopathology, without clinical evidence of pulmonary vasculopathy, sufficient? How best to define the presence of pulmonary vasculopathy is a particularly interesting question, given that studies that defined it by PFT (24), imaging (26), or histopathology (50) all suggest that its presence is associated with worse outcomes.

Beyond a recognition that changes to the initial criteria are needed, more fundamental questions about the natural history and prognosis of IPAF remain unanswered. Do patients go on to develop a defined CTD, or is IPAF a distinct disease entity with pulmonary involvement as the primary manifestation? Does the interstitial pneumonia with IPAF behave like that of CTD-ILD or more like IIP? Is it the underlying lung injury pattern that predicts prognosis or treatment responsiveness, or are there specific autoimmune clinical, serologic, or morphologic features that impact prognostically and therapeutically?

Put more simply, should patients with IPAF be treated similarly to those with CTD-ILD by using immunosuppressive therapies, or should they be treated with an antifibrotic similar to IPF? Should we base such decisions on underlying lung injury patterns and/or presence of fibrosis? We await subset analysis from the phase II clinical trial with pirfenidone for unclassifiable ILD, as a subset of the enrolled subjects fulfill IPAF criteria (clinicaltrials. gov identifier: NCT03099187) (51). These and, we hope, other prospective, randomized controlled clinical trials will augment our understanding of how to manage patients with IPAF. 
Finally, although intended as a research classification, we anticipate that over time there will be evolution of IPAF to a clinical diagnosis - or at least a provisional oneand perhaps IPAF will be represented in the ILD classification schema as a distinct subset residing in the intersection between IIP and CTD-ILD.

\section{Conclusions}

The construct of IPAF represents an important first step in standardizing the nomenclature and providing uniform classification criteria that establish a platform for research of patients with IIP and features suggestive of autoimmunity. A research classification or "diagnosis" of IPAF may be provisional, as some patients may evolve to characterizable forms of CTD-ILD. Initial studies of patients identified by retrospective application of the IPAF criteria demonstrate that there is substantial heterogeneity among these cohorts, and these data highlight a need for refinement of the criteria, particularly with more structure and uniformity of how to define the morphologic domain. Future research is needed to determine whether the natural history of IPAF is influenced by specific phenotypic clinical, serologic, or morphologic features for prognostication and to identify effective therapeutic approaches to help in the care of these patients.

Author disclosures are available with the text of this article at www.atsjournals.org.

Acknowledgment: Dr. Graney thanks the Reuben M. Cherniak Fellowship Award at National Jewish Health for providing ongoing support of her research.

\section{References}

1 Raghu G, Remy-Jardin M, Myers JL, Richeldi L, Ryerson CJ, Lederer DJ, et al.; American Thoracic Society, European Respiratory Society, Japanese Respiratory Society, and Latin American Thoracic Society. Diagnosis of idiopathic pulmonary fibrosis: an Official ATS/ERS/JRS/ ALAT clinical practice guideline. Am J Respir Crit Care Med 2018;198: e44-e68.

2 Lee CT, Oldham JM. Interstitial pneumonia with autoimmune features: overview of proposed criteria and recent cohort characterization. Clin Pulm Med 2017;24:191-196.

3 Corte TJ, Copley SJ, Desai SR, Zappala CJ, Hansell DM, Nicholson AG, et al. Significance of connective tissue disease features in idiopathic interstitial pneumonia. Eur Respir J 2012;39:661-668.

4 Fischer A, du Bois R. Interstitial lung disease in connective tissue disorders. Lancet 2012;380:689-698.

5 Cottin V. Significance of connective tissue diseases features in pulmonary fibrosis. Eur Respir Rev 2013;22:273-280.

6 Collins BF, Spiekerman CF, Shaw MA, Ho LA, Hayes J, Spada CA, et al. Idiopathic interstitial pneumonia associated with autoantibodies: a large case series followed over 1 year. Chest 2017;152:103-112.

7 Fischer A, West SG, Swigris JJ, Brown KK, du Bois RM. Connective tissue disease-associated interstitial lung disease: a call for clarification. Chest 2010;138:251-256.

8 Kinder BW, Collard HR, Koth L, Daikh DI, Wolters PJ, Elicker B, et al. Idiopathic nonspecific interstitial pneumonia: lung manifestation of undifferentiated connective tissue disease? Am J Respir Crit Care Med 2007;176:691-697.

9 Vij R, Noth I, Strek ME. Autoimmune-featured interstitial lung disease: a distinct entity. Chest 2011;140:1292-1299.

10 Assayag D, Kim EJ, Elicker BM, Jones KD, Golden JA, King TE Jr, et al. Survival in interstitial pneumonia with features of autoimmune disease: a comparison of proposed criteria. Respir Med 2015;109: 1326-1331.

11 Fischer A, Antoniou KM, Brown KK, Cadranel J, Corte TJ, du Bois RM, et al.; ERS/ATS Task Force on Undifferentiated Forms of CTD-ILD. An official European Respiratory Society/American Thoracic Society research statement: interstitial pneumonia with autoimmune features. Eur Respir J 2015;46:976-987.

12 Ramos-Casals M, García-Carrasco M, Brito MP, López-Soto A, Font J. Autoimmunity and geriatrics: clinical significance of autoimmune manifestations in the elderly. Lupus 2003;12:341-355.

13 Lee JS, Kim EJ, Lynch KL, Elicker B, Ryerson CJ, Katsumoto TR, et al. Prevalence and clinical significance of circulating autoantibodies in idiopathic pulmonary fibrosis. Respir Med 2013;107:249-255.

14 Nagai S, Handa T, Tabuena R, Kitaichi M, Izumi T. Nonspecific interstitial pneumonia: a real clinical entity? Clin Chest Med 2004;25:705-715, vi.

15 Spagnolo P, Lee JS, Sverzellati N, Rossi G, Cottin V. The Lung in rheumatoid arthritis: focus on interstitial lung disease. Arthritis Rheumatol 2018;70:1544-1554.
16 Doyle TJ, Dellaripa PF. Lung manifestations in the rheumatic diseases. Chest 2017;152:1283-1295.

17 Tansey D, Wells AU, Colby TV, Ip S, Nikolakoupolou A, du Bois RM, et al. Variations in histological patterns of interstitial pneumonia between connective tissue disorders and their relationship to prognosis. Histopathology 2004;44:585-596.

18 Elliot TL, Lynch DA, Newell JD Jr, Cool C, Tuder R, Markopoulou K, et al. High-resolution computed tomography features of nonspecific interstitial pneumonia and usual interstitial pneumonia. $J$ Comput Assist Tomogr 2005;29:339-345.

19 Hobbs S, Lynch D. The idiopathic interstitial pneumonias: an update and review. Radiol Clin North Am 2014;52:105-120.

20 Hunninghake GW, Lynch DA, Galvin JR, Gross BH, Müller N, Schwartz $\mathrm{DA}$, et al. Radiologic findings are strongly associated with a pathologic diagnosis of usual interstitial pneumonia. Chest 2003;124:1215-1223.

21 Chung JH, Chawla A, Peljto AL, Cool CD, Groshong SD, Talbert JL, et al CT scan findings of probable usual interstitial pneumonitis have a high predictive value for histologic usual interstitial pneumonitis. Chest 2015;147:450-459.

22 Gruden JF, Panse PM, Leslie KO, Tazelaar HD, Colby TV. UIP diagnosed at surgical lung biopsy, 2000-2009: HRCT patterns and proposed classification system. AJR Am J Roentgenol 2013;200:W458-W467.

23 Fischer A, Lee JS, Cottin V. Interstitial lung disease evaluation: detecting connective tissue disease. Respiration 2015;90:177-184.

24 Oldham JM, Adegunsoye A, Valenzi E, Lee C, Witt L, Chen L, et al. Characterisation of patients with interstitial pneumonia with autoimmune features. Eur Respir J 2016;47:1767-1775.

25 Ley B, Ryerson CJ, Vittinghoff E, Ryu JH, Tomassetti S, Lee JS, et al. A multidimensional index and staging system for idiopathic pulmonary fibrosis. Ann Intern Med 2012;156:684-691.

26 Chung JH, Montner SM, Adegunsoye A, Lee C, Oldham JM, Husain AN, et al. CT findings, radiologic-pathologic correlation, and imaging predictors of survival for patients with interstitial pneumonia with autoimmune features. AJR Am J Roentgenol 2017;208:1229-1236.

27 Chartrand S, Swigris JJ, Stanchev L, Lee JS, Brown KK, Fischer A. Clinical features and natural history of interstitial pneumonia with autoimmune features: a single center experience. Respir Med 2016; 119:150-154.

28 Chartrand S, Lee JS, Fischer A. Longitudinal assessment of interstitia pneumonia with autoimmune features is encouraged. Respir Med 2017;132:267.

29 Ahmad K, Barba T, Gamondes D, Ginoux M, Khouatra C, Spagnolo P, et al. Interstitial pneumonia with autoimmune features: clinical, radiologic, and histological characteristics and outcome in a series of 57 patients. Respir Med 2017;123:56-62.

30 Cutolo M, Pizzorni C, Sulli A, Smith V. Early diagnostic and predictive value of capillaroscopy in systemic sclerosis. Curr Rheumatol Rev 2013;9:249-253.

31 Ito Y, Arita M, Kumagai S, Takei R, Noyama M, Tokioka F, et al. Serological and morphological prognostic factors in patients with interstitial pneumonia with autoimmune features. BMC Pulm Med 2017;17:111. 
32 Dai J, Wang L, Yan X, Li H, Zhou K, He J, et al. Clinical features, risk factors, and outcomes of patients with interstitial pneumonia with autoimmune features: a population-based study. Clin Rheumatol 2018;37:2125-2132.

33 Yoshimura K, Kono M, Enomoto $\mathrm{Y}$, Nishimoto $\mathrm{K}$, Oyama $\mathrm{Y}$, Yasui $\mathrm{H}$, et al. Distinctive characteristics and prognostic significance of interstitial pneumonia with autoimmune features in patients with chronic fibrosing interstitial pneumonia. Respir Med 2018;137:167-175.

34 Kelly BT, Moua T. Overlap of interstitial pneumonia with autoimmune features with undifferentiated connective tissue disease and contribution of UIP to mortality. Respirology 2018;23:600-605.

35 Solomon JJ, Chung JH, Cosgrove GP, Demoruelle MK, FernandezPerez ER, Fischer A, et al. Predictors of mortality in rheumatoid arthritis-associated interstitial lung disease. Eur Respir J 2016;47: 588-596.

36 Park JH, Kim DS, Park IN, Jang SJ, Kitaichi M, Nicholson AG, et al. Prognosis of fibrotic interstitial pneumonia: idiopathic versus collagen vascular disease-related subtypes. Am J Respir Crit Care Med 2007; 175:705-711.

37 Cavagna L, Gonzalez Gay MA, Allanore Y, Matucci-Cerinic M. Interstitial pneumonia with autoimmune features: a new classification still on the move. Eur Respir Rev 2018;27:30.

38 Jee AS, Bleasel JF, Adelstein S, Keir GJ, Corte TJ. A call for uniformity in implementing the IPAF (interstitial pneumonia with autoimmune features) criteria. Eur Respir J 2016;48:1811-1813.

39 Jee AS, Adelstein S, Bleasel J, Keir GJ, Nguyen M, Sahhar J, et al. Role of autoantibodies in the diagnosis of connective-tissue disease ILD (CTD-ILD) and interstitial pneumonia with autoimmune features (IPAF). J Clin Med 2017;6:E51.

40 Hozumi H, Enomoto N, Oyama Y, Kono M, Fujisawa T, Inui N, et al. Clinical implication of proteinase-3-antineutrophil cytoplasmic antibody in patients with idiopathic interstitial pneumonias. Hai 2016; 194:235-242.

41 Hozumi H, Oyama $\mathrm{Y}$, Yasui H, Suzuki Y, Kono M, Karayama M, et al. Clinical significance of myeloperoxidase-anti-neutrophil cytoplasmic antibody in idiopathic interstitial pneumonias. PLoS One 2018;13: e0199659.

42 Lundberg IE, Tjärnlund A, Bottai M, Werth VP, Pilkington C, de Visser M, et al.; International Myositis Classification Criteria Project Consortium, the Euromyositis Register, and the Juvenile Dermatomyositis Cohort
Biomarker Study and Repository (UK and Ireland). 2017 European League against Rheumatism/American College of Rheumatology classification criteria for adult and juvenile idiopathic inflammatory myopathies and their major subgroups. Arthritis Rheumatol 2017;69: 2271-2282.

43 Collins B, Raghu G. Interstitial pneumonia with autoimmune features: the new consensus-based definition for this cohort of patients should be broadened. Eur Respir J 2016;47:1293-1295.

44 Aggarwal R, McBurney C, Schneider F, Yousem SA, Gibson KF, Lindell $\mathrm{K}$, et al. Myositis-associated usual interstitial pneumonia has a better survival than idiopathic pulmonary fibrosis. Rheumatology (Oxford) 2017;56:384-389.

45 Moua T, Zamora Martinez AC, Baqir M, Vassallo R, Limper AH, Ryu JH. Predictors of diagnosis and survival in idiopathic pulmonary fibrosis and connective tissue disease-related usual interstitial pneumonia. Respir Res 2014;15:154.

46 Strand MJ, Sprunger D, Cosgrove GP, Fernandez-Perez ER, Frankel SK, Huie TJ, et al. Pulmonary function and survival in idiopathic vs secondary usual interstitial pneumonia. Chest 2014;146:775-785.

47 Song JW, Lee HK, Lee CK, Chae EJ, Jang SJ, Colby TV, et al. Clinical course and outcome of rheumatoid arthritis-related usual interstitial pneumonia. Sarcoidosis Vasc Diffuse Lung Dis 2013;30:103-112.

48 Richeldi L, du Bois RM, Raghu G, Azuma A, Brown KK, Costabel U, et al.; INPULSIS Trial Investigators. Efficacy and safety of nintedanib in idiopathic pulmonary fibrosis. N Engl J Med 2014;370:2071-2082.

49 King TE Jr, Bradford WZ, Castro-Bernardini S, Fagan EA, Glaspole I, Glassberg MK, et al.; ASCEND Study Group. A phase 3 trial of pirfenidone in patients with idiopathic pulmonary fibrosis. $N$ Engl $J$ Med 2014;370:2083-2092.

50 Adegunsoye A, Oldham JM, Valenzi E, Lee C, Witt LJ, Chen L, et al. Interstitial pneumonia with autoimmune features: value of histopathology. Arch Pathol Lab Med 2017;141:960-969.

51 Maher TM, Corte TJ, Fischer A, Kreuter M, Lederer DJ, Molina-Molina M, et al. Pirfenidone in patients with unclassifiable progressive fibrosing interstitial lung disease: design of a double-blind, randomised, placebo-controlled phase II trial. BMJ Open Respir Res 2018;5: e000289.

52 Ryerson CJ, Collard HR. Update on the diagnosis and classification of ILD. Curr Opin Pulm Med 2013;19:453-459. 